\title{
Vancomycin Heteroresistance in vanM-type Enterococcus faecium
}

\author{
Ying Zhou, ${ }^{1,2, *}$ Yang Yang, ${ }^{1,2,{ }^{\star}}$ Li Ding, ${ }^{1,2}$ Chunhui Chen, ${ }^{3}$ Xiaogang $X u^{1,2,4}$ and Minggui Wang ${ }^{1,2,4}$
}

\begin{abstract}
Although vancomycin heteroresistance exists in enterococci, it is not easily detected using routine methods and the mechanism is not clearly understood. In this study, we characterized the molecular mechanism underlying vancomycin heteroresistance in a clinical vanM-type Enterococcus faecium strain. The original E. faecium isolate, hVREm7, was susceptible to vancomycin by broth microdilution and Etest. However, vancomycinresistant subcolonies were present within the Etest zone of inhibition. Three passages of hVREm7 were carried out to eliminate the possibility of contamination. hVREm7 and three resistant variants were selected to study the heteroresistance mechanism. Sequence analysis revealed that all four strains contained an unaltered vanM cluster. Southern blot analysis showed that vanM was present both chromosomally and extrachromosomally in the three variants, but only extrachromosomally in hVREm7. The size of the vanM-bearing extrachromosomal DNA fragments in the three variants was larger than that in hVREm7, indicating a variation of vanM gene amplification in the variants. Consistently, vanM copy number and expression level were increased in variant strain VREm7-1. These results suggest that partial or complete amplification/transfer of the vanM gene cluster by an as-yet-unidentified mechanism leads to increased copy number and augmented expression of the vanM gene, which contribute to the transformation of vancomycin-heteroresistant E. faecium into high-level vancomycin-resistant variants.
\end{abstract}

Keywords: heteroresistance, vancomycin, Enterococcus faecium, vanM, copy number

\section{Introduction}

QINCE FIRST ENCOUNTERED in 1986, vancomycin$\checkmark$ resistant enterococci (VRE) have emerged as important nosocomial pathogens and become a global public health concern. $^{1-4}$ In the United States, there are 54,500 health care-associated infections caused by VRE, resulting in 5,400 deaths in $2017 .{ }^{5}$ Although the prevalence of VRE remains relatively low in mainland China, incidence levels have been increasing over the past decades. ${ }^{6}$ To date, nine types of vancomycin resistance genotypes (vanA, vanB, vanC, vanD, vanE, van $G$, vanL, vanM, and vanN) have been identified in enterococci, with the vanA and vanB genotypes predominating worldwide. ${ }^{7}$ The relatively uncommon clinical vanM-type vancomycin-resistant Enterococcus faecium (VREm) strain was first isolated from a hospital in Shanghai, China, in $2006 .^{8}$ vanM-type VREm has been the predominant type in Shanghai hospitals since $2011 .^{9}$ A recent study reported 143 vanM-carrying $E$. faecium isolates from an infected patient and the environmental surfaces of an intensive care unit in Hangzhou, a city near Shanghai. ${ }^{10}$ vanM-type VREm was also identified in Singapore in 2011. ${ }^{11}$ The emergence and spread of vanM-type VREm are of great concern.

In clinical microbiology laboratories, VRE isolates are usually detected based on traditional testing methods such as automated antimicrobial susceptibility testing. However, some bacteria show classical heteroresistance; the majority of the bacterial population is susceptible to an antibiotic, while the minority is highly resistant. ${ }^{12}$ Traditional testing methods often fail to detect vancomycin-heteroresistant strains. ${ }^{12}$ Vancomycin heteroresistance in enterococci was first detected in a vanA-type E. faecium isolate from a patient in 2001. ${ }^{13}$ Mutations or insertions in the van gene cluster can lead to heteroresistance among VRE strains under continuous exposure to vancomycin. ${ }^{14,15}$ However, the precise mechanism underlying vancomycin heteroresistance in enterococci remains unclear. In this study, we report a clinical vanM-type

\footnotetext{
${ }^{1}$ Institute of Antibiotics, Huashan Hospital, Fudan University, Shanghai, China.

${ }^{2}$ Key Laboratory of Clinical Pharmacology of Antibiotics, National Health Commission, Shanghai, China.

${ }^{3}$ Division of Infectious Diseases, Massachusetts General Hospital, Boston, Massachusetts, USA.

${ }^{4}$ National Clinical Research Center for Aging and Medicine, Huashan Hospital, Fudan University, Shanghai, China.

*These authors contributed equally to this work.
} 
vancomycin-heteroresistant $E$. faecium strain and vanM gene cluster amplification/transfer by an as-yet-unidentified mechanism, which leads to elevated copy numbers and expression levels of the vanM gene, as well as a high-level vancomycin resistance.

\section{Materials and Methods}

\section{Bacterial strains}

The original clinical strain, hVREm7, was isolated from the urine of a patient who had not received vancomycin treatment at a tertiary care hospital in Shanghai, China, in 2016. The organism was identified using the VITEK 2 Compact microbial identification system (bioMérieux, France). The original isolate was susceptible to vancomycin when tested using the broth microdilution method. However, when this isolate was subjected to the Etest (bioMérieux) assay, some subcolonies were observed within the zone of inhibition (Fig. 1), suggesting that hVREm7 was heteroresistant to vancomycin. To confirm that the original strain was vancomycin heteroresistant, rather than a mixture of resistant and nonresistant bacteria, the isolate was daily streaked on plates with or without vancomycin for 3 days (Supplementary Fig. S1). The hVREm7 isolate showed a classical form of heteroresistance (the majority of the population was susceptible to vancomycin, with a highly resistant minority) during three passages (Supplementary Fig. S1). These results confirmed that the hVREm7 isolate was van-

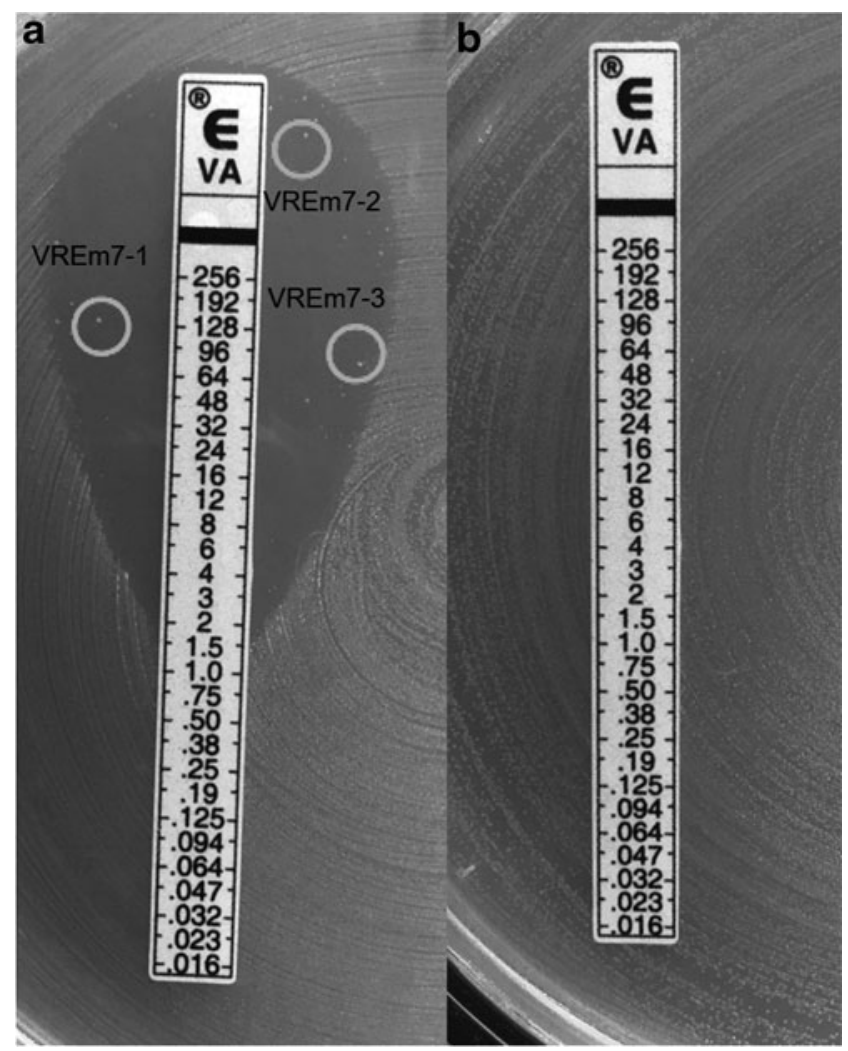

FIG. 1. Etest results for vancomycin susceptibility in (a) hVREm7 and (b) VREm-7-1 (VREm7-2 and VREm7-3 showed the same results as VREm7-1). VREm, vancomycinresistant Enterococcus faecium. comycin heteroresistant and excluded the possibility of contamination. To study the heteroresistance mechanism, three colonies within the zone of inhibition, VREm7-1, VREm7-2, and VREm7-3, were selected for further analysis (Fig. 1).

\section{Antimicrobial susceptibility testing}

The vancomycin minimum inhibitory concentrations (MICs) of the original isolate and the three variants were determined by both broth microdilution and Etest methods following the Clinical and Laboratory Standards Institute guidelines. ${ }^{16,17}$ Vancomycin MIC determination was also repeated using the VITEK 2 Compact system. Susceptibility testing for teicoplanin, linezolid, penicillin, nitrofurantoin, ampicillin, tigecycline, erythromycin, levofloxacin, chloromycetin, and rifampin was performed using the broth microdilution method. ${ }^{16,17}$ Enterococcus faecalis ATCC 29212 was used as a quality control strain for MIC determination and the original vanM-type VREm strain, Efm-HS0661, and vanA-type VREm strain, BM4147, ${ }^{18}$ were used as positive polymerase chain reaction (PCR) controls.

\section{Analysis of vancomycin resistance genes and the vanM gene cluster}

The presence of van genes in the original isolate and its three variants was determined by PCR and sequencing with primers NvanF and NvanR (listed in Supplementary Table S1). ${ }^{8}$ The structure of the vanM gene cluster was determined using a PCR-mapping assay with primers (Supplementary Table S1) derived from the sequence of reference strain Efm-HS0661 (van $M$ type). ${ }^{8}$

\section{Pulsed-field gel electrophoresis and multilocus sequence typing analysis}

Pulsed-field gel electrophoresis (PFGE) plugs of strain $\mathrm{hVREm} 7$ and its three variants were prepared as previously described. ${ }^{9}$ DNA was left undigested or digested with the restriction enzyme SmaI (TaKaRa, Dalian, China). PFGE analysis was performed with a CHEF mapper system (Bio$\mathrm{Rad})$. The digested and undigested DNA fragments were separated for $22 \mathrm{hr}$ at $6 \mathrm{~V} / \mathrm{cm}, 14^{\circ} \mathrm{C}$ on a $1.0 \%$ agarose gel (Bio-Rad) with pulse times of 5-30 sec. XbaI-digested Salmonella Braenderup H9812 DNA was used as the DNA marker. The nucleic acid dye GelGreen (Tiangen, China) was used to stain the DNA in the gels. Band patterns were analyzed as per the criteria devised by Tenover et al. ${ }^{19}$ Multilocus sequence typing (MLST) was performed by sequencing partial fragments of seven housekeeping genes (atpA, ddl, gdh, purk, gyd, pstS, and $a d k)$. These nucleotide sequences were compared to preexisting sequences in the MLST database (http://efaecium.mlst.net/) to determine the alleles and sequence types (STs) of the E. faecium isolates.

\section{Southern blotting}

Southern blotting was performed to determine the sizes of the DNA fragment(s) harboring the vanM genes in the clinical isolate and its variants. Following electrophoresis, the undigested DNA fragments were transferred to a positively charged nylon membrane (Roche Diagnostics, Germany) using a capillary transfer system for $18 \mathrm{hr}$. The vanM gene and 16s rRNA gene (to distinguish the location of 
chromosome and plasmids) probe were prepared using primers vanM-probe F/R and 16s rRNA-probe F/R (Supplementary Table S1) and labeled with digoxigenin using a PCR DIG probe synthesis kit (Roche Diagnostics). The membrane was then hybridized with the digoxigenin-labeled vanM probe following the manufacturer's protocols. Hybridization signals were detected using a ChemiDoc XRS+ imaging system (Bio Rad).

\section{Determination of gene copy number and gene expression}

Quantitative PCR (qPCR) was carried out to detect the copy numbers of vanM in hVREm7, the VREm7-1 variant, and Efm-HS0061. All strains were induced with vancomycin $(20 \mathrm{mg} / \mathrm{L})$ for $30 \mathrm{~min}$ before DNA and RNA extractions. Reverse transcription PCR (RT-PCR) was performed to evaluate the relative gene expression levels of the vanM gene. The DNA samples were prepared using the TIANamp Bacteria DNA Kit (Tiangen) and the RNA samples were extracted using the MiniBEST Universal RNA Extraction Kit (TaKaRa). Total RNA was purified after eliminating genomic DNA with gDNA Eraser (TaKaRa) and then reverse transcribed into cDNA using the PrimeScriptRT reagent Kit. qPCR was performed using a ViiA 7 system (Applied Biosystems, CA). A $20 \mu \mathrm{L}$ PCR reaction mixture was prepared with $10 \mu \mathrm{L} 2 \times \mathrm{SYBR}$ Green Master Mix (TaKaRa), $1.6 \mu \mathrm{L} 10 \mu \mathrm{M}$ primers, $6 \mu \mathrm{L}$ UltraPure $\mathrm{H}_{2} \mathrm{O}$, $0.4 \mu \mathrm{L} 50 \times$ ROX Reference Dye II, and $2 \mu \mathrm{L}$ cDNA. PCR cycling conditions were $95^{\circ} \mathrm{C}$ for $30 \mathrm{sec}, 40$ cycles of $95^{\circ} \mathrm{C}$ for $5 \mathrm{sec}$, and $60^{\circ} \mathrm{C}$ for $34 \mathrm{sec}$, followed by the melt curve setting of 1 cycle of $95^{\circ} \mathrm{C}$ for $15 \mathrm{sec}, 60^{\circ} \mathrm{C}$ for $1 \mathrm{~min}$, and $95^{\circ} \mathrm{C}$ for $15 \mathrm{sec}$. The E. faecium housekeeping gene atpA was used as an internal control for both DNA and RNA qualification. The gene copy numbers and gene expressions of the hVREm7 were used as the control group; the other experimental groups will use this as a reference to calculate the relative fold changes. The relative expression levels of van $M$ were calculated using the $\triangle \Delta C T$ method. All assays were carried out in triplicate with three independent biological DNA and RNA preparations. The difference in relative expression was analyzed by Student's $t$-test with Bonferroni correction.

\section{Statistics}

Statistical significance was assessed using a two-tailed Student's $t$-test, of the GraphPad Prism8 software. $p<0.05$ was considered statistically significant.

\section{Results}

Antimicrobial susceptibility of hVREm7 and its three variants

The vancomycin MIC of the original clinical isolate (hVREm7) was $1 \mathrm{mg} / \mathrm{L}$, as determined by broth microdilution after $24 \mathrm{hr}$ of incubation and the same by VITEK2. By Etest, the vancomycin MIC of hVREm7 was $2 \mathrm{mg} / \mathrm{L}$, with a subpopulation of resistant colonies within the zone of inhibition (Fig. 1a). The vancomycin MICs of the three subcolonies VREm7-1, VREm7-2, and VREm7-3, were $128 \mathrm{mg} / \mathrm{L}$ by broth microdilution and $>256 \mathrm{mg} / \mathrm{L}$ by Etest. Notably, when measured after $48 \mathrm{hr}$ of incubation, the hVREm 7 could grow in the broth containing higher vancomycin concentrations $(64 \mathrm{mg} / \mathrm{L}, \mathrm{MIC}=128 \mathrm{mg} / \mathrm{L})$ and so did three subcolonies (from 64 to $128 \mathrm{mg} / \mathrm{L}, \mathrm{MIC}=256 \mathrm{mg} / \mathrm{L}$ ). In contrast, the vancomycin MICs of the control strains, E. faecium EfmHS0661 (vanM type) and BM4147 (vanA type), remained the same at $24 \mathrm{hr}$ and $48 \mathrm{hr}$ (Tables 1 and 2). The teicoplanin MICs of the hVREm7 isolate and its three variants were 1 and $64 \mathrm{mg} / \mathrm{L}$, respectively, as determined by broth microdilution after $24 \mathrm{hr}$ of incubation. After $48 \mathrm{hr}$, the teicoplanin resistance level of hVREm7 and the three subcolonies was increased, which allowed them to grow in higher concentrations of teicoplanin (Table 2). No significant difference was observed between the original isolate and its three variants for the MICs of the other antimicrobial agents tested.

\section{Molecular characteristics}

The original isolate hVREm7 and its variants, VREm71, VREm7-2, and VREm7-3, were vanM positive by PCR amplification and sequencing using generic primers for van genes. ${ }^{8}$ PCR mapping and sequencing showed that all four vanM gene clusters were flanked by two direct IS 1216 repeats, as reported in Efm-HSO661 ${ }^{8}$; no point mutations or insertions were detected in the vanM gene cluster based on a comparison between parental strain hVREm7 and the three subcolonies. In addition, MLST typing demonstrated that all four strains had the same ST, ST262, which is designated as $\mathrm{CC} 17$ by the eBURST algorithm. SmaI digestion PFGE analysis also revealed the same pattern for all four strains (Fig. 2a). These results suggest that these four strains belong to the same clone, further eliminating the possibility of mixed cultures.

Although there was no difference in molecular structure between the four strains, all had a 109-bp deletion in the IS1216 transposases (which is adjacent to vanRM element) and the vanRM intergenic region compared with the prototype vanM element (detected in Efm-HS0661; Supplementary Fig. S2). In addition, Neural Network Promoter Prediction $^{20}$ results indicated that this deletion generated a

TABle 1. VANCOMYCin Minimum Inhibitory Concentrations of Various Strains Tested WITH VITEK2, ETEST, AND MicrodiLUtion

\begin{tabular}{lcccc}
\hline & \multicolumn{4}{c}{ Vancomycin MIC (mg/L) } \\
\cline { 2 - 5 } Strain & VITEK2 & Etest & Time $(\mathrm{hr})$ & Microdilution $^{\mathrm{a}}$ \\
\hline hVREm7 & 1 & 2 & 24 & 1 \\
VREm7-1 & $\geq 32$ & $>256$ & 24 & 128 \\
& & & 48 & 256 \\
VREm7-2 & $\geq 32$ & $>256$ & 24 & 128 \\
VREm7-3 & $\geq 32$ & $>256$ & 24 & 256 \\
& & & 48 & 128 \\
Efm-HS0661 & $\geq 32$ & $>256$ & 24 & 256 \\
& & & 48 & 256 \\
BM4147 & $\geq 32$ & $>256$ & 24 & 256 \\
& & & 48 & 256 \\
\hline
\end{tabular}

${ }^{\mathrm{a}}$ Vancomycin MICs of various strains obtained after incubation of 24 and $48 \mathrm{hr}$ in the microdilution test.

MICs, minimum inhibitory concentrations; VREm, vancomycinresistant Enterococcus faecium. 
Table 2. Antimicrobial Susceptibility of hVREm7 and Its Subcolonies by Broth Microdilution

\begin{tabular}{|c|c|c|c|c|c|c|c|c|c|c|c|}
\hline \multirow[b]{2}{*}{ Strains } & \multicolumn{11}{|c|}{$M I C(m g / L)$} \\
\hline & $V A N$ & $T E C$ & $L N Z$ & $T G C$ & $P E N$ & $A M P$ & NIT & $L V X$ & $E R Y$ & $R I F$ & CHL \\
\hline \multicolumn{12}{|l|}{$24 \mathrm{hr}$} \\
\hline hVREm7 & 1 & 1 & 1 & 0.125 & $>128$ & $>128$ & 64 & 64 & $>32$ & 4 & 16 \\
\hline VREm7-1 & 128 & 64 & 1 & 0.125 & $>128$ & $>128$ & 128 & 64 & $>32$ & 2 & 16 \\
\hline VREm7-2 & 128 & 64 & 1 & 0.125 & $>128$ & $>128$ & 128 & 64 & $>32$ & 2 & 16 \\
\hline VREm7-3 & 128 & 64 & 1 & 0.125 & $>128$ & $>128$ & 128 & 64 & $>32$ & 4 & 16 \\
\hline \multicolumn{12}{|l|}{$48 \mathrm{hr}$} \\
\hline hVREm7 & 128 & 32 & 1 & 0.125 & $>128$ & $>128$ & 64 & 64 & $>32$ & 4 & 16 \\
\hline VREm7-1 & 256 & 128 & 1 & 0.125 & $>128$ & $>128$ & 128 & 64 & $>32$ & 2 & 16 \\
\hline VREm7-2 & 256 & 128 & 1 & 0.125 & $>128$ & $>128$ & 128 & 64 & $>32$ & 2 & 16 \\
\hline VREm7-3 & 256 & 128 & 1 & 0.125 & $>128$ & $>128$ & 128 & 64 & $>32$ & 4 & 16 \\
\hline
\end{tabular}

AMP, ampicillin; CHL, chloromycetin; ERY, erythromycin; LNZ, linezolid; LVX, levofloxacin; NIT, nitrofurantoin; PEN, penicillin; RIF, rifampicin; TEC, teicoplanin; TGC, tigecycline; VAN, vancomycin.

mutation in the vanRM promoter, which may play a role in the expression of the $v a n M$ gene cluster, as part of a twocomponent regulatory system that senses vancomycin. ${ }^{8} \mathrm{~A}$ promoter activity assay was used to evaluate the possible effect of this deletion on $v a n R M$ promoter function. In contrast with our hypothesis, the mutation had no significant effect on promoter functionality (data not shown).

The underlying mechanism was further investigated using the PFGE assay with hVREm7 and its variants. PFGE anal- ysis identified variance among the four strains. Although the sizes of the DNA fragments could not be accurately determined by undigested PFGE analysis, owing to different migration of linear and circular DNA, the extrachromosomal DNA fragments of the three variants were larger compared with hVREm7 (Fig. 2b). The digoxigenin-labeled 16s rRNA probe was used to distinguish the chromosomal and extrachromosomal fragments more clearly. Southern hybridization analysis with the $16 \mathrm{~s} r N A$ probe accurately exposed the a

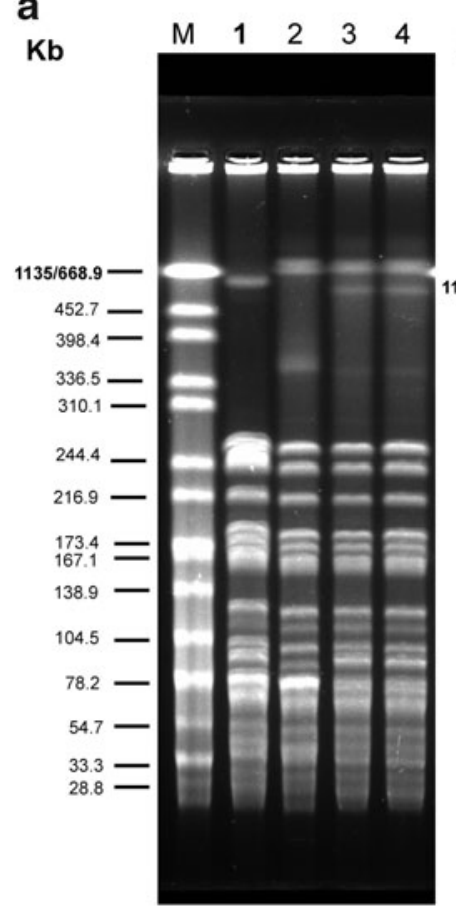

b Kb
C

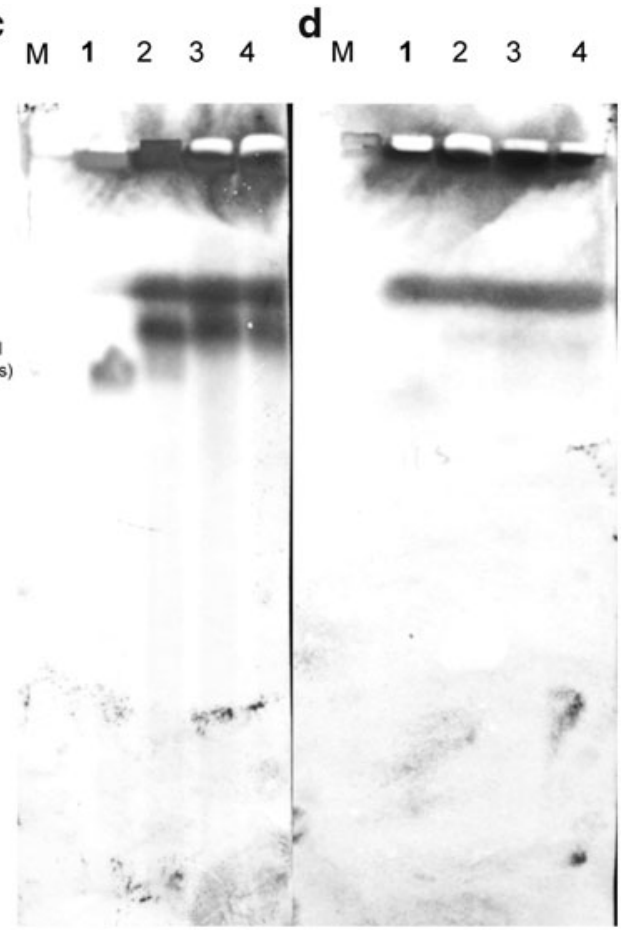

FIG. 2. PFGE of genomic DNA and Southern hybridization profiles of hVREm7 and its three variants. (a) SmaI digestion profile of total DNA: lane M, marker (XbaI-digested DNA of Salmonella Braenderup H9812); lanes 1-4, hVREM7 and its three variants: VREm7-1, VREm7-2, and VREm7-3. (b) Undigested PFGE profile of total DNA: lane M, marker (H9812); lanes 1-4, hVREM7 and its three variants VREm7-1, VREm7-2, and VREm7-3; lane NC (negative control), the blank gel with wrap no genomes. (c) Southern blot with digoxigenin-labeled vanM gene-specific probe: lane M, marker (H9812); lanes 1-4, hVREM7 and its three variants VREm7-1, VREm7-2, and VREm7-3. (d) Southern blot with digoxigenin-labeled 16S rRNA gene-specific probe: lane M, marker (H9812); lanes 1-4, hVREM7 and its three variants VREm7-1, VREm7-2, and VREm7-3. PFGE, pulsed-field gel electrophoresis. 
location of the chromosomal fragments in four isolates (Fig. 2d). Furthermore, Southern hybridization analysis with a digoxigenin-labeled $v a n M$ probe revealed the presence of vanM genes in both the chromosomal and extrachromosomal DNA of the three variants, but only in the extrachromosomal DNA of the original isolate. Notably, the sizes of the vanMbearing extrachromosomal DNA fragments of the three variants were larger compared with their parental strain (Fig. 2c). We hypothesized that more than one copy of the vanM gene might be present in the variants.

\section{vanM gene copy number and gene expression}

qPCR was carried out to evaluate the copy number and relative expression of the $v a n M$ and $v a n R M$ genes in the original isolate (hVREm7) and one representative variant (VREm7-1). The qPCR results showed that the copy numbers of both the vanM and vanRM genes were significantly higher in VREm7-1 than in hVREm7 (Fig. 3a). In agreement with the copy number results, the abundance of vanM and vanRM mRNA was also significantly different; vanM and vanRM transcript levels were $>20$-fold higher in the variant compared with the original isolate hVREm7 (Fig. 3b).

The aforementioned results illustrate the hVREm7 remained susceptible to vancomycin regardless of the presence of the vanM gene cluster. After eliminating the impact of the promoter mutation, we hypothesized that the low copy numbers in the original isolate affected susceptibility. Accordingly, the same qPCR assay was conducted for hVREm7 and prototype strain Efm-HS0661; the copy numbers and relative expression were normalized to Efm-HS0661. As shown in the bar charts in Fig. 3, both vanM/RM copy numbers and transcript levels were considerably lower in $\mathrm{hVREm} 7$ than in the prototype isolate.

\section{Discussion}

There are many reports about hetero-vancomycinresistance in staphylococci, ${ }^{12,21}$ but rare in enterococci. This may be due to a failure to detect the heteroresistant population by standard susceptibility testing methods. ${ }^{22}$ Population analysis profiling (PAP) is the gold standard for the identification of heteroresistance; however, this is a labor-intensive process not suitable for clinical microbiology laboratories. $^{12,22}$ The Etest assay, in which the growth of discrete colonies within the zone of inhibition indicates heteroresistance, is an alternative method to PAP. ${ }^{12,22}$ In our study, the original clinical isolate hVREm7 was sensitive to vancomycin when tested using a standardized automated system (VITEK 2 Compact) and broth microdilution method. However, a vancomycin-resistant subpopulation of hVREm7 was observed within the vancomycin Etest zone of inhibition. PFGE analysis revealed that the original clinical isolate hVREm7 and the three selected subcolonies (VREm7-1, VREm7-2, and VREm7-3) belong to the same clone, which eliminated the possibility of contamination. In addition, all four strains were positive for vanM, as determined by PCR and sequencing. Because of the difficulty in detection, the prevalence of vancomycin-heteroresistant vanM-type enterococci in Shanghai remains unknown and requires further investigation.

Despite being detected since $2001,{ }^{13}$ studies on vancomycin heteroresistance in enterococci are rare and the heteroresistance mechanism is not yet fully understood. ${ }^{14,15}$ To the best of our knowledge, this is the first report of a vanMtype vancomycin-heteroresistant $E$. faecium isolate. The original isolate, hVREm7, was susceptible to both vancomycin and teicoplanin, as determined by three different methods after $24 \mathrm{hr}$ of incubation. Notably, when the susceptibility results were read after $48 \mathrm{hr}$, both the vancomycin and teicoplanin MICs for hVREm7 increased to a resistant level. These results suggest that glycopeptide resistance in the hVREm7 strain was augmented under antibiotic exposure, consistent with the results of former studies. ${ }^{13}$ Vancomycinpromoted resistance has been observed in both enterococcal strains $^{14,15}$ and Staphylococcus aureus. ${ }^{23}$ Several studies have also demonstrated that heteroresistance is a common resistance phenotype among clinical isolates, which would have been missed based on standard MIC estimates. ${ }^{22}$

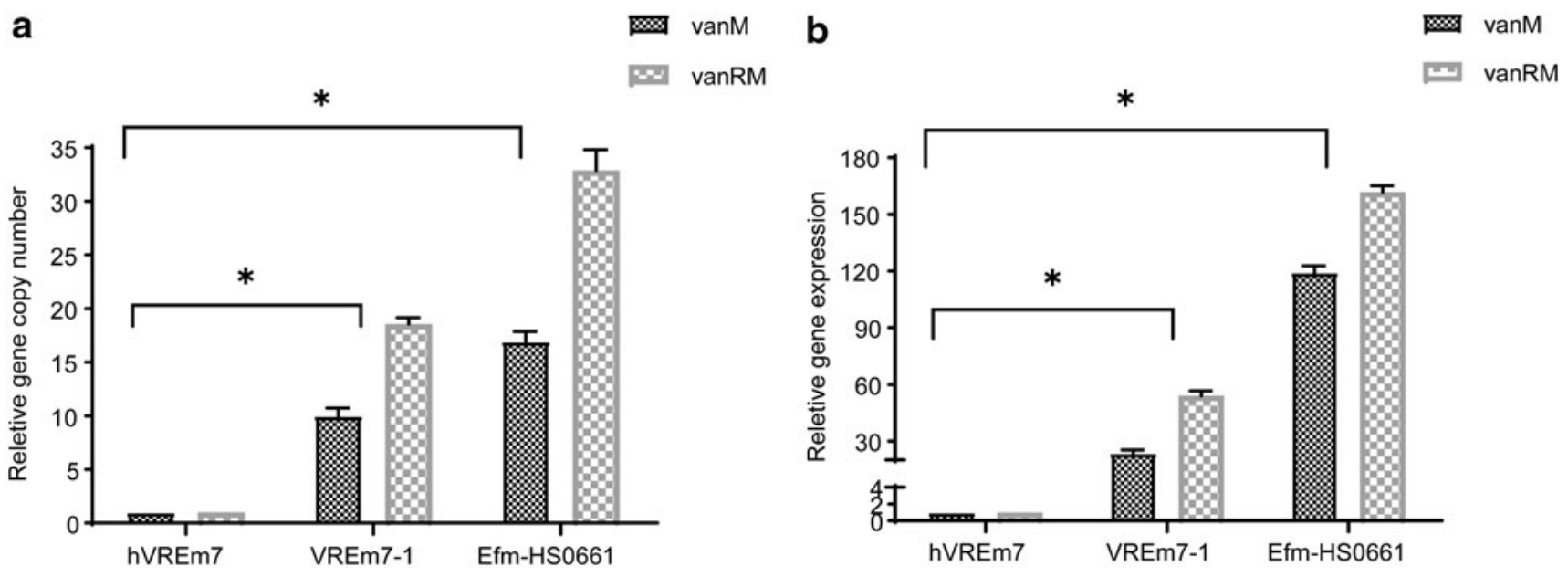

FIG. 3. Relative $v a n M$ and $v a n R M$ copy number and gene expression in selected isolates. (a) Relative $v a n M / R M$ gene copy number in VREm7-1, and Efm-HS0661 compared with hVREm7. (b) Relative vanM/RM gene expression level in VREm7-1, and Efm-HS0661 compared with hVREm7. The data represent the mean \pm standard deviation for three independent biological replicates. Differences between different strains, in regard to copy number or gene expression of both vanM and $v a n R M$ genes, were statistically analyzed using a two-tailed Student's $t$-test with Bonferroni correction. ${ }^{*} p<0.05$. 
Although the specific clinical prevalence of heteroresistance is unknown, it is possible that more resistant subpopulations may be selected during antimicrobial therapy, which could lead to treatment failure and persistent infection. ${ }^{21,23}$ Therefore, it is important to routinely screen for heteroresistance if vancomycin is used alone in treatment of a persistent enterococcal infection such as bacteremia.

Heteroresistance in bacterial populations has been associated with genetic mechanisms such as mutations, gene duplication, or epigenetic variation. ${ }^{12}$ Mutations or insertions in the vanA gene have been shown to be responsible for vancomycin heteroresistance in vanA-type E. faecium. ${ }^{14,15}$ In our study, we did not detect any mutation or insertion in the vanM gene cluster when examining the parental strain and the three subcolonies; however, compared with the prototype vanM gene cluster, all four isolates had a 109-bp deletion in the IS1216 transposases and the vanRM intergenic region. Interestingly, this deletion had no significant effect on the function of the vanM gene cluster; although both hVREm7 and its variants possessed the same deletion, hVREm7 remained susceptible to vancomycin, despite the presence of the vanM gene cluster.

Notably, the vanM gene is located on extrachromosomal DNA in clinical strain hVREm7, while in the three variants, the vanM gene is present on both chromosomal and extrachromosomal DNA. Moreover, the size of the vanM-bearing region in the three variants was larger than that in their parental strain. To identify the potential mechanism, we determined the copy numbers and expression levels of the $v a n M / R M$ genes in one variant and parental strain hVREm7; both the copy numbers and expression levels were higher in the resistant variant than in the original strain, hVREm7. Excluding the molecular structure differences between hVREm 7 and its variants, the increased copy number and expression level of the vanM gene cluster might contribute to the high-level vancomycin resistance in the variants. Further analysis of copy number and expression levels of vanM and vanRM using corresponding Efm-HS0661 genes as references confirmed our hypothesis that $\mathrm{hVREm} 7$ is a specific isolate harboring a low copy number and expression of the $v a n M$ gene.

The qPCR tests demonstrated that gene expression was positively correlated with copy number, in agreement with previous findings that tandem amplification of resistance genes is a general mechanism for heteroresistance. ${ }^{22}$ However, point mutations or small deletions associated with some host regulators also mediate resistance alteration between mutated and ancestral isolates. ${ }^{22}$ This may explain the relatively higher mRNA transcript level compared with the copy number of the same elements; other, unidentified regulatory variations may also exist, but we did not find these at this stage.

Tandem amplification of resistance genes is the most common mechanism for heteroresistance and amplification breakpoints are usually located within directly repeated sequences such as transposases or insertion sequences (IS) for amplifications on plasmids. ${ }^{22}$ As we observed, the vanM gene cluster in the four strains was flanked by two directly oriented copies of IS1216. The IS1216-like element belongs to the IS26 family, which has played a pivotal role in the dissemination of resistance determinants in Gram-positive bacteria. ${ }^{24}$ IS1216 movement was previously demonstrated to occur by replicative transposition, which results in a cointegrate of the donor and recipient molecules with a directly repeated copy of the IS at each junction, creating a composite transposon-like structure. $^{24}$ This may explain how a small vanM-carrying plasmid flanked by two directly oriented copies of IS1216 was incorporated into large plasmids or the chromosome, as shown in Fig. 2 (Supplementary Fig. S3). ${ }^{24}$ Very similar IS26mediated amplification of resistance genes have been described in VIM-1-producing Klebsiella pneumoniae strains; the IncN plasmid that mediates the higher imipenem MIC carries two copies of the In-541 integron containing the $b l a_{\mathrm{VIM}-1}$ gene flanked by IS26 elements. ${ }^{25}$ Furthermore, a recent geneticsbased study showed that plasmid recombination is attributed to the loss of vancomycin resistance following vancomycin withdrawal. ${ }^{26}$ It has also been shown that the chromosome position affects gene expression profiles in bacteria. ${ }^{27}$ Considering these findings, we hypothesize that the vanM gene cluster might insert in both chromosomal DNA and plasmids owing to recombination events, resulting in multiple copies of the vanM gene with increased expression. Whole-genome sequencing might confirm the existence of multiple copies of vanM and reveal their exact location.

In conclusion, we report a vanM-type vancomycinheteroresistant E. faecium isolate. Partial or complete amplification/transfer of the vanM gene cluster by an as-yetundetermined mechanism leads to an increased copy number and augmented expression of the vanM gene, which contribute to vancomycin-heteroresistant E. faecium transforming into high-level vancomycin-resistant variants. These potential vancomycin resistance change observed in this study could lead to treatment failure and persistent infection, calling for the development of facile and rapid protocols to identify heteroresistance in these pathogens.

\section{Acknowledgment}

We thank Dr. George A. Jacoby (Lahey Clinic, Burlington, Massachusetts) for his critical review of the manuscript.

\section{Disclosure Statement}

No competing financial interests exist.

\section{Funding Information}

This study was supported by grants from the National Natural Science Foundation of China (81171613, 81971978 to X.X.).

\section{Supplementary Material}

Supplementary Figure S1

Supplementary Figure S2

Supplementary Figure S3

Supplementary Table S1

\section{References}

1. Fisher, K., and C. Phillips. 2009. The ecology, epidemiology and virulence of Enterococcus. Microbiology 155: 1749-1757.

2. Gilmore, M.S., F. Lebreton, and W. van Schaik. 2013. Genomic transition of enterococci from gut commensals to leading causes of multidrug-resistant hospital infection in the antibiotic era. Curr. Opin. Microbiol. 16:10-16.

3. Neelakanta, A., S. Sharma, V.P. Kesani, M. Salim, A. Pervaiz, N. Aftab, T. Mann, N. Tashtoush, S. Karino, S. 
Dhar, and K.S. Kaye. 2015. Impact of changes in the NHSN catheter-associated urinary tract infection (CAUTI) surveillance criteria on the frequency and epidemiology of CAUTI in intensive care units (ICUs). Infect. Control Hosp. Epidemiol. 36:346-349.

4. Leclercq, R., E. Derlot, J. Duval, and P. Courvalin. 1988. Plasmid-mediated resistance to vancomycin and teicoplanin in Enterococcus faecium. N. Engl. J. Med. 319:157-161.

5. CDC's Antibiotic Resistance Threats in the United States, 2019. Centers for Disease Control and Prevention, US Department of Health and Human Services. https://www.cdc.gov/ drugresistance/biggest-threats.html\#van (accessed January 13, 2020).

6. Zhao, C., H. Sun, H. Wang, Y. Liu, B. Hu, Y. Yu, Z. Sun, Y. Chu, B. Cao, K. Liao, J. Lei, Z. Hu, L. Zhang, X. Zhang, Y. Xu, Z. Wang, and M. Chen. 2012. Antimicrobial resistance trends among 5608 clinical Gram-positive isolates in China: results from the Gram-Positive Cocci Resistance Surveillance program (2005-2010). Diagn. Microbiol. Infect. Dis. 73:174-181.

7. Cattoir, V., and R. Leclercq. 2013. Twenty-five years of shared life with vancomycin-resistant enterococci: is it time to divorce? J. Antimicrob. Chemother. 68:731-742.

8. Xu, X., D., Lin, G. Yan, X. Ye, S. Wu, Y. Guo, D. Zhu, F. Hu, Y. Zhang, F. Wang, G.A. Jacoby, and M. Wang. 2010. vanM, a new glycopeptide resistance gene cluster found in Enterococcus faecium. Antimicrob. Agents Chemother. 54: 4643-4647.

9. Chen, C., J. Sun, Y. Guo, D. Lin, Q. Guo, F. Hu, D. Zhu, X. $\mathrm{Xu}$, and $\mathrm{M}$. Wang. 2015. High prevalence of vanM in vancomycin-resistant Enterococcus faecium isolates from Shanghai, China. Antimicrob. Agents Chemother. 59: 7795-7798.

10. Zhang, G., X. Lin, J. Cai, R. Zhang, H. Xiu, B. Tian, and W. Cui. 2018. Characteristics of clinical and environmental vanM-carrying vancomycin-resistant enterococci isolates from an infected patient. Int. J. Antimicrob. Agents. 51: 932-935.

11. Teo, J.W., P. Krishnan, R. Jureen, and R.T. Lin. 2011. Detection of an unusual van genotype in a vancomycinresistant Enterococcus faecium hospital isolate. J. Clin. Microbiol. 49:4297-4298.

12. El-Halfawy, O.M., and M.A. Valvano. 2015. Antimicrobial heteroresistance: an emerging field in need of clarity. Clin. Microbiol. Rev. 28:191-207.

13. Alam, M.R., S. Donabedian, W. Brown, J. Gordon, J.W. Chow, M.J. Zervos, and E. Hershberger. 2001. Heteroresistance to vancomycin in Enterococcus faecium. J. Clin. Microbiol. 39:3379-3381.

14. Khan, S.A., K. Sung, S. Layton, and M.S. Nawaz. 2008. Heteroresistance to vancomycin and novel point mutations in Tn1546 of Enterococcus faecium ATCC 51559. Int. J. Antimicrob. Agents 31:27-36.

15. Park, I.J., W.G. Lee, J.H. Shin, K.W. Lee, and G.J. Woo. 2008. VanB phenotype-vanA genotype Enterococcus faecium with heterogeneous expression of teicoplanin resistance. J. Clin. Microbiol. 46:3091-3093.

16. Clinical and Laboratory Standards Institute. 2018. Methods for Dilution Antimicrobial Susceptibility Tests for Bacteria That Grow Aerobically. 11th ed. CLSI M07. Clinical and Laboratory Standards Institute, Wayne, PA.

17. Clinical and Laboratory Standards Institute. 2018. Performance Standards for Antimicrobial Susceptibility Testing. 28th ed. CLSI M100-M128. Clinical and Laboratory Standards Institute, Wayne, PA.

18. Arthur, M., C. Molinas, F. Depardieu, and P. Courvalin. 1993. Characterization of Tn1546, a Tn3-related transposon conferring glycopeptide resistance by synthesis of depsipeptide peptidoglycan precursors in Enterococcus faecium BM4147. J. Bacteriol. 175:117-127.

19. Tenover, F.C., R.D. Arbeit, R.V. Goering, P.A. Mickelsen, B.E. Murray, D.H. Persing, and B. Swaminathan. 1995. Interpreting chromosomal DNA restriction patterns produced by pulsed-field gel electrophoresis: criteria for bacterial strain typing. J. Clin. Microbiol. 33:2233-2239.

20. Reese, M.G. 2001. Application of a time-delay neural network to promoter annotation in the Drosophila melanogaster genome. Comput. Chem. 26:51-56.

21. Hiramatsu, K. 2001. Vancomycin-resistant Staphylococcus aureus: a new model of antibiotic resistance. Lancet Infect. Dis. 1:147-155.

22. Nicoloff, H., K. Hjort, B.R. Levin, and D.I. Andersson. 2019. The high prevalence of antibiotic heteroresistance in pathogenic bacteria is mainly caused by gene amplification. Nat. Microbiol. 4:504-514.

23. Sakoulas, G., J. Alder, C. Thauvin-Eliopoulos, R.C. Moellering, Jr., and G.M. Eliopoulos. 2006. Induction of daptomycin heterogeneous susceptibility in Staphylococcus aureus by exposure to vancomycin. Antimicrob. Agents Chemother. 50:1581-1585.

24. Partridge, S.R., S.M. Kwong, N. Firth, and S.O. Jensen. 2018. Mobile genetic elements associated with antimicrobial resistance. Clin. Microbiol. Rev. 31:e00088-17.

25. Loli, A., L.S. Tzouvelekis, E. Tzelepi, A. Carattoli, A.C. Vatopoulos, P.T. Tassios, and V. Miriagou. 2006. Sources of diversity of carbapenem resistance levels in Klebsiella pneumoniae carrying blaVIM-1. J. Antimicrob. Chemother. 58:669-672.

26. Chacko, K.I., M.J. Sullivan, C. Beckford, D.R. Altman, B. Ciferri, T.R. Pak, R. Serba, A. Kasarskis, C.L. Hamula, and H. van Bakel. 2018. Genetic basis of emerging vancomycin, linezolid, and daptomycin heteroresistance in a case of persistent Enterococcus faecium bacteremia. Antimicrob. Agents Chemother. 62:e02007-17.

27. Bryant, J.A., L.E. Sellars, S.J. Busby, and D.J. Lee. 2014. Chromosome position effects on gene expression in Escherichia coli K-12. Nucleic Acids Res. 42:11383-11392.

Address correspondence to: Xiaogang $X u, M D$

Institute of Antibiotics Huashan Hospital Fudan University 12 M. Wulumuqi Road Shanghai 200040 China

E-mail: xuxiaogang@fudan.edu.cn

Chunhui Chen, MD, PhD Division of Infectious Diseases Massachusetts General Hospital 55 Fruit Street Boston, MA 02114 USA

E-mail: cchen49@partners.org 